\title{
First considerations on the Balance of Plant for a HELIAS fusion power plant
}

\author{
Felix Warmer ${ }^{\mathrm{a}, *}$, Evaldas Bubelis ${ }^{\mathrm{b}}$ \\ ${ }^{a}$ Max Planck Institute for Plasma Physics, D-17491, Greifswald, Germany \\ ${ }^{b}$ Karlsruhe Institute of Technology, Institute for Neutron Physics and Reactor Technology, 76344 Eggenstein-Leopoldshafen, Germany
}

\begin{abstract}
A first conceptual design of the Balance of Plant for a HELIAS power plant equipped with a Helium Cooled Pebble Bed (HCPB) Breeder Blanket (BB) is presented in this work. 5 inboard and 10 outboard cooling loops extract the heat from the blanket. Heat exchangers are used to transfer the thermal power from the helium to solar salt, which transports the heat out of the stellarator building and into an industrial 2 -tank thermal energy storage system (IHTS/ESS). For electricity generation, the heat from the solar salt is transferred to the feedwater, which is boiled and superheated in the steam generator and then used in the turbine.

In absence of a detailed design for the HCPB BB in stellarator geometry and the ex-vessel piping, a sensitivity study was carried out for the pressure drop of the cooling loops which showed that even small changes in the pressure drop have significant impact on the required pumping power.

Finally, an exemplary runtime scenario is presented demonstrating the capability of a stellarator fusion power plant equipped with an IHTS/ESS to operate very flexibly $( \pm 20 \%)$ in an envisaged dynamic future energy system. Considering demand forecasts and the ESS, a stellarator fusion power plant (FPP) seems well equipped to cope with fluctuating grid demands within its technical limitations. If the conceptual idea for the HELIAS BoP as presented in this paper can be confirmed by more detailed technical studies, then a stellarator FPP could potentially contribute to grid stabilization in a future energy system while replacing fossil fuel power plants as a sustainable energy source.
\end{abstract}

Keywords: HELIAS, Balance of Plant (BoP), Energy storage system (ESS), Helium Cooled Pebble Bed (HCPB)

\section{Introduction}

Nuclear fusion by magnetic confinement is considered to be a sustainable, $\mathrm{CO}_{2}$-free energy source and therefore offers the prospect of easing the energy demand of mankind by the end 5 of this century and beyond. The leading concepts for magnetic ${ }_{35}$ confinement are the tokamak and the stellarator. So far, the breadth of fusion research has focused on the development of the core components of these devices, i.e. the plasma confinement, superconducting coils, divertor, blanket, etc. in order to 10 solve the known tokamak DEMO issues regarding plasma stability and control, low availability, as well as high recirculating electrical power. While these issues do not exist in a stellarator, the common non-device specific issues like the fuel cycle, material qualification, and cost still apply. However, all these 15 issues are strongly addressed in the European fusion research programme [1.

Assuming that these issues can be solved and that fusion power plants will be introduced to the market and grid by the end of this century, it follows that fusion must fit a future en-

20 ergy system which is envisaged to be a multi-modular, decentral, and dynamic system with significant contributions from ${ }^{50}$ fluctuating energy sources - as is, for example, foreseen by the German 'Energiewende'.

Consequently, research needs to be initiated on how fusion 25 energy can be successfully and commercially integrated into a complex energy system as expected in 2050 and beyond. This includes socio-economic and technical aspects, as well as advanced runtime scenarios. An important technical aspect is thereby the interface between the fusion core, which produces

\footnotetext{
${ }^{*}$ Corresponding author, Tel.: +49 (0)3834 88-1738

Email address: Felix.Warmer@ipp.mpg.de (Felix Warmer)
}

30 the high-grade heat, and the demand side of the electrical grid. This interface is the so-called 'Balance of Plant' (BoP) which essentially encompasses all power flows within, as well as in and out of the power plant. The BoP is the sum of all components which are responsible for heat and power transfer, power storage, as well as power conversion. Thus, it is clear that the outline and design of the Balance of Plant is a keystone for achieving a successful and efficient integration of fusion energy into the future energy system.

This fact has already been recognised by the German Helmholtz Programme Nuclear Fusion and the European Fusion Programme (EUROfusion) and has lead to a first conceptual design of the Balance of Plant systems for the tokamak EU Demonstration Fusion Reactor (DEMO) 2. It should be noted that the BoP design and outline of its components depends on the employed Breeding Blanket (BB) concept. Among many details, the $\mathrm{BB}$ concept determines the type of the primary coolant (i.e. pressurised water or gaseous helium), the associated pressure drop along the pipes and the available temperature range which are important inputs for e.g. the dimensioning of the primary heat-exchangers and compressors. Currently, there are several different BB concepts under consideration around the world. One of the most promising BB concepts, which is extensively studied in Europe is the so-called Helium Cooled Pebble Bed (HCPB). The HCPB consists of ${ }^{6} \mathrm{Li}$ enriched pebbles for tritium breeding, Be as neutron multiplier, EUROFER as structural material, and helium as coolant [3]. Due to its favourable properties, the HCPB has also been recently used as basis for the study of the Balance of Plant for a DEMO device [2] and is consequently also used as reference BB for the study presented in this paper.

Since tokamaks operate intrinsically in a pulsed mode (unless substantial non-inductive current drive is provided), DEMO 
could only provide electricity in pulsed operation. However, this limitation could be overcome by introduction of a solar salt 65 thermal storage in the DEMO BoP design 2]. With such an internal storage, the dwell time can be bridged by accumulating heat during the pulse period and releasing it during the dwell ${ }_{12}$ period essentially allowing DEMO to run at $80 \%$ nominal power in steady-state.

70 Considering these achievements, in this work we present a first conceptual design for the Balance of Plant of a helical-axis advanced stellarator (HELIAS) type fusion power plant 4, 5].130 The outline of the conceptual HELIAS BoP is presented in section 2. Associated quasi-steady-state simulations are shown in 75 section 3 . including a sensitivity study of the coolant pressure drop as well as demonstrating a flexible runtime scenario suitable for a dynamic future energy system. Finally, the work is 135 summarised in section 4 with some remarks on the outlook.

\section{HELIAS Balance of Plant Design}

The conceptual design of the HELIAS Balance of Plant, pre- ${ }^{140}$ sented here, is based on the initial considerations done for the tokamak DEMO using the HCPB BB 2, 6, 77. The BoP can be categorised in terms of its subsystems. These are the Primary Heat Transfer System (PHTS) for the BB, the Intermedi-

85 ate Heat Transfer System (IHTS) including a thermal Energy Storage System (ESS) as well as the Power Conversion System (PCS). This chain of systems is conceptually illustrated in Fig. 1

In general, the blanket concept has direct impact on the $90 \mathrm{BoP}$ and the PHTS since the BB design determines the type of coolant and the in- and outlet temperatures. Similarly to the DEMO study, in this work the HCPB concept is used as a first step for the design of the HELIAS BoP (with helium as primary coolant). As the HCPB exhibits a large global energy 95 multiplication factor $\left(M_{E}=1.35\right)$ 8, the total thermal power considered for the study here is $3840 \mathrm{MW}$ assuming $3000 \mathrm{MW}$ fusion power 9 .

\subsection{Primary Heat Transfer System}

The current HELIAS concept is a large aspect ratio stellarator consisting of five identical field periods [5, 10, 11]. From a design point of view, it is thus meaningful that the BB heat transfer system follows this symmetry. Further boundary conditions are given by the distribution of the thermal power ${ }_{150}$ within the stellarator. I.e., about $1 / 3$ of the thermal power goes to the inboard (IB) side and the remaining $2 / 3$ to the outboard (OB) side. Consequently, we chose a design with a total of 15 independent heat transfer loops: 5 for the IB side and 10 for the OB side ( 1 IB and 2 OB loops per field period). ${ }_{15}$ Such a design fulfills both the HELIAS symmetry and follows

110 the thermal power distribution. Further, each loop has its own intermediate heat exchanger (IHX) and for safety reasons two blowers in order to reduce risks from a partial Loss Of Flow Accident (LOFA) in case of a blower failure. Moreover, the segmentation and separation of the independent cooling loops serves the purpose of limiting consequences from Loss Of Cooling Accidents (LOCA) as well as to reduce the size of the main components (IHX, piping, compressors).

For simplicity and in absence of a detailed design, all BB heat transfer loops are assumed to be identical. Thus, each helium transferred to the solar salt within the IHX. With this dimensioning of the systems, the loops and IHX are comparable to the tokamak DEMO IB heat transfer loops. It should be noted that the pressure drop along the pipes depends very much on the details of the design. Since a full systems design is beyond the scope of this work, we assume here the same pressure drop for the helium loops and the IHX as was calculated for DEMO with $\Delta p_{\text {loop }}=2.6$ bar for each loop and $\Delta p_{\text {IHX }}=0.8$ bar for each IHX. Based on these values, the total required pumping power for the helium compressors is calculated to be on the order of $P_{\text {pump }} \approx 220$ MW. While most of the system is commercially available, the IHX and compressors are quite large needing further optimisation in collaboration with industry.

Apart from the main PHTS system which extracts the heat from the BB, the PHTS includes also three smaller systems which collect low-grade heat from the divertor plasma facing components (DIV-PFC), divertor cassette (DIV-Cas), and the vacuum vessel (VV). The main parameters of these components are shown in Tab. 1

The low-grade heat from the DIV-PFC, DIV-Cas and VV is used to pre-heat the feedwater on the return line from the condenser to the steam generator. Each of these three heat transfer systems has its own IHX and water pump. In order to cope with the different temperature requirements for all three systems, the feedwater flow around the three IHXs is split into a main flow and a by-pass flow.

\begin{tabular}{|c|c|c|c|c|}
\hline Heat Source & $\begin{array}{l}\text { BB } \\
\text { Helium }\end{array}$ & $\begin{array}{l}\text { DIV-Cas } \\
\text { Water }\end{array}$ & $\begin{array}{l}\text { DIV-Pfc } \\
\text { Water }\end{array}$ & $\begin{array}{l}\text { VV } \\
\text { Water }\end{array}$ \\
\hline $\begin{array}{l}\text { Thermal } \\
\text { Power [MW] }\end{array}$ & 3598 & 80 & 132 & 30 \\
\hline $\begin{array}{l}\text { Total mass } \\
\text { flow rate }[\mathrm{kg} / \mathrm{s}]\end{array}$ & 3470 & 598 & 5319 & 673 \\
\hline $\begin{array}{l}\text { Outlet } \\
\text { temperature }\left[{ }^{\circ} \mathrm{C}\right]\end{array}$ & 500 & 210 & 136 & 200 \\
\hline $\begin{array}{l}\text { Inlet } \\
\text { temperature }\left[{ }^{\circ} \mathrm{C}\right]\end{array}$ & 300 & 180 & 130 & 190 \\
\hline $\begin{array}{l}\text { Operation } \\
\text { pressure [bar] }\end{array}$ & 80 & 35 & 50 & 31.5 \\
\hline
\end{tabular}

Table 1: Design values for the HELIAS PHTS. The values for the Thermal Power are taken from [9].

\subsection{Intermediate Heat Transfer System}

The IHTS consists of solar salt and corresponding tanks to store a sensible amount of heat at sufficiently high temperatures. The storage used in this work is an existing industrial 2-tank design which is commonly used in today's concentrated solar power plants (CSP) 12. The main design parameters of IHTS are shown in Tab. 2 It should be noted, however, that studies are ongoing to replace this system by a more efficient 1-tank thermocline system in the near future.

\begin{tabular}{ll}
\hline Coolant & Solar Salt \\
\hline Solar salt mass $[\mathrm{kt}]$ & $\sim 40$ \\
Hot tank temperature $\left[{ }^{\circ} \mathrm{C}\right]$ & 480 \\
Cold tank temperature $\left[{ }^{\circ} \mathrm{C}\right]$ & 268 \\
Operational pressure $[\mathrm{bar}]$ & 1.0 \\
\hline
\end{tabular}

Table 2: Design values for the HELIAS IHTS/ESS.

Originally, the IHTS/ESS was introduced for the pulsed tokamak DEMO in order to buffer sufficient heat during the pulse time to bridge the electricity generation during the dwell time. This approach ensures a continuous operation of the 
PHTS

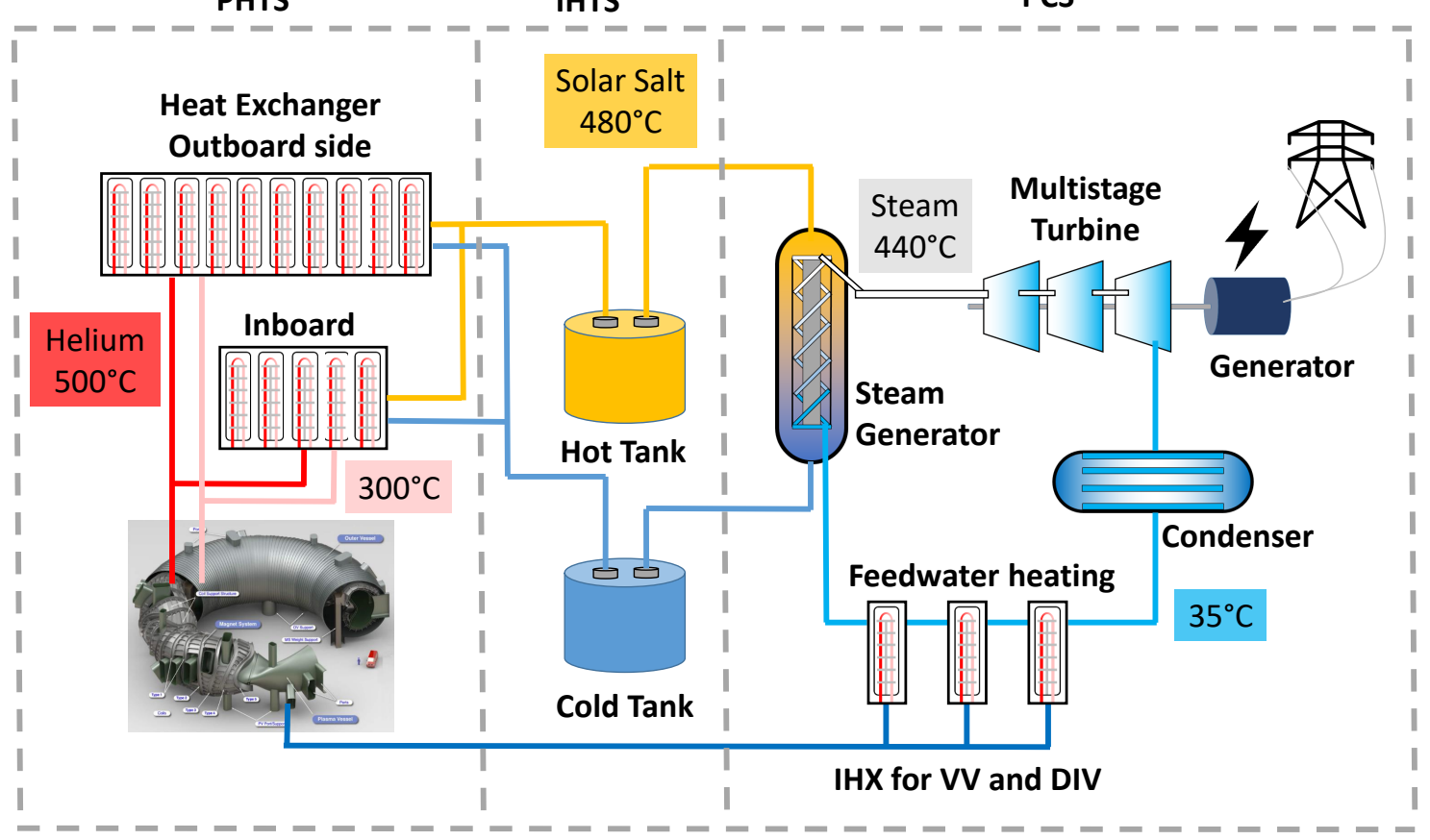

Figure 1: Schematic outline of the HELIAS Balance of Plant with a HCPB Breeder Blanket.

DEMO PCS system allowing a steady-state electricity generation at about $80 \%$ nominal power.

Stellarators are by design steady-state capable, however with the drawback that the plasma performance is optimised for a single fixed operation point with fixed fusion power output. This would mean that a stellarator could only run at $100 \%$ power capacity without any flexibility. However, with an integrated IHTS/ESS, this limitation can be overcome. During a low demand phase, a part of the heat can be stored in the ESS. Thus, the reactor core continues to run at $100 \%$ capacity while the net electric output to the grid is reduced. Then, in an high demand phase, additional electricity can be provided to the grid by additionally converting the stored heat in the ${ }_{195}$ ESS to electricity on top of the normal operation of the reactor core. Thus, within the limitations of the turbine and the ESS storage capacity, the fixed power output of a stellarator can be overcome resulting in a desired flexibility of net power to the grid. While operation at $90-110 \%$ nominal power is available ${ }_{200}$ with such a scheme by default, the goal should be to reach $80-120 \%$ in order to cope with the requirements of a dynamic future energy system. A possible flexible runtime scenario will be presented in subsection 3.2 .

\subsection{Power Conversion System}

The conceptual design of the PCS is based on a classical water-steam Rankine cycle operating at about 58 bar with superheated steam at $\sim 440^{\circ} \mathrm{C}$. The cycle consists of a steam generator, reheater as well as a set of low pressure (LP) and high pressure (HP) turbine stages. As discussed before, a part of the feedwater is pre-heated by the heat transfer system from the DIV-PFC, DIV-Cas and VV. The main parameters of the 210 HELIAS PCS are given in Tab. 3

With this conversion cycle a gross efficiency of about $\eta_{\text {gross }}=$ $38-39 \%$ is reached (ratio of gross electrical power to total ther-

\begin{tabular}{ll}
\hline Coolant & Water / Steam \\
\hline Gross power [MW] & 1482 \\
Net power [MW] & 1240 \\
Gross efficiency [\%] & 38.4 \\
Net efficiency [\%] & 32.6 \\
Inlet HP turbine temp. $\left[{ }^{\circ} \mathrm{C}\right]$ & 438.5 \\
Outlet HP turbine temp. $\left[{ }^{\circ} \mathrm{C}\right]$ & 170 \\
Inlet LP turbine temp. $\left[{ }^{\circ} \mathrm{C}\right]$ & 279 \\
Outlet LP turbine temp. $\left[{ }^{\circ} \mathrm{C}\right]$ & 35.5 \\
\hline
\end{tabular}

Table 3: Design values for the HELIAS PCS.

mal power). However, with helium as primary coolant, significant power $(\sim 220 \mathrm{MW})$ needs to be recirculated for the helium pumping, leading to a net efficiency of about $\eta_{\text {net }}=32-33 \%$. Other sources for recirculating power have been neglected here, which will further reduce the net efficiency.

It should be noted that the temperature of the solar salt returning to the cold tank needs to be kept stable and sufficiently high to avoid salt freezing $\left(\sim 220^{\circ} \mathrm{C}\right)$. The chosen temperature is $268^{\circ} \mathrm{C}$, which is also needed to keep the temperature of the helium returning back to the $\mathrm{BB}$ at $300^{\circ} \mathrm{C}$. Generally, it is important that the operating temperatures of all systems are consistent with each other and are kept stable during operation.

Here, the temperatures are mostly limited by the envisaged materials. For example, the temperatures of the PHTS heat transfer loops $\left(300^{\circ} \mathrm{C}\right.$ in, $500^{\circ} \mathrm{C}$ out $)$ are determined by the limitations of the structural material, in this case EUROFER.

\section{Simulation of HELIAS Balance-of-Plant}

For the simulation of the HELIAS BoP, the industrial software package EBSILON $\cap$ has been used [13]. EBSILON $\cap$ encompasses a database of pre-defined components (turbines, heat exchanger, etc.) from which a thermodynamic power 
plant cycle can be modeled. For the simulation of such cy-

EBSILONR iteratively solves the underlying equations of all components providing a static or quasi-static solution to the255 whole system. This allows one to easily obtain a comprehensive map of all thermo-hydraulic parameters for all components and pipes. Therefore, EBSILON@ is generally used and well suited for the design and optimisation of all kinds of power plant cycles.

\subsection{Sensitivity Study of Pressure Drop}

As already discussed for the PHTS in subsection 2.1, the pressure drop along the heat transfer loops depends on the details of the actual piping and blanket design. However, such a design task is currently beyond the scope of this work which concentrates on an initially simplified, yet holistic system view. As the considered heat transfer loop outline for the HELIAS in this work is similar to the DEMO IB loops, the pressure drop calculated for DEMO has also here been assumed as a nominal value $\left(\Delta p_{\text {tot }}=3.4\right.$ bar for each loop).

Since the pressure drop has a strong impact on the required pumping power for the helium compressors, a sensitivity study was carried out by systematically varying the pressure drop in the simulation model around the nominal value. The results of this study are illustrated in Fig. 2

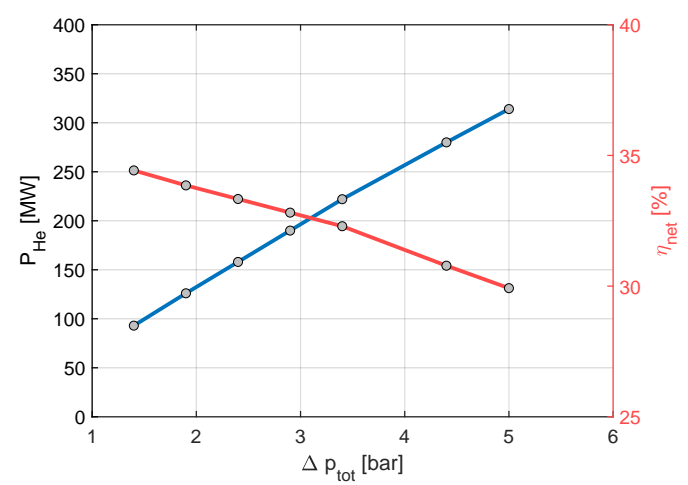

Figure 2: Required helium pumping power as function of total pressure drop (blue line, left axis) as well as resulting net efficiency of ${ }^{275}$ the BoP cycle $\eta_{\text {net }}$ (orange line, right axis).

It can be seen that already small to moderate changes in the pressure drop have a significant impact on the required pumping power. For the HCPB, the pumping power is the main contribution to the recirculating power and thus impacts the net plant efficiency. In order to determine and minimise the pressure drop, a detailed design of the HCPB in HELIAS geometry is necessary with an outline for the ex-vessel piping and IHXs.

\subsection{Flexible Runtime Scenario}

As already discussed in the introduction and subsection 2.2 a future fusion power plant may need to adapt to a complex and dynamic energy system. In order to be able to react in a timely manner to low or high demand from the grid, a fusion power plant needs to retain sufficient flexibility. For a HELIAS 290 power plant which is designed for a fixed fusion power output, this flexibility is realised by means of the IHTS/ESS. This can be illustrated by the exemplary runtime scenario shown in Fig. 3 which is simulated with EBSILON@ in a quasi-static mode by means of a time series.

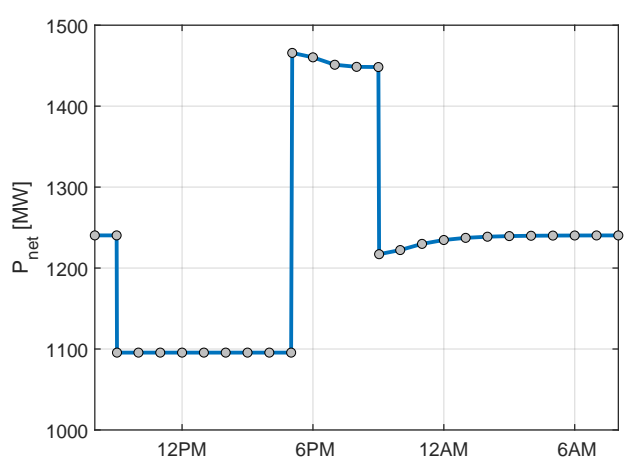

Figure 3: Net electric power for a HELIAS power plant as function of time for a flexible runtime scenario.

Starting from the nominal net electric power of about $1250 \mathrm{MW}$, the electrical power output to the grid is reduced to $90 \%$, corresponding to $1100 \mathrm{MW}$. The excess heat is used to charge the ESS accumulating 34453 tons of hot solar salt during an 8 hour low demand phase phase. This stored heat can then be used to provide additional electricity to the grid during an expected (or unexpected) high demand time. In such a case, the nominal power output is increased to $120 \%$ corresponding to $1450 \mathrm{MW}$ which could be realised for a total of 4 hours given accumulated heat. It should be noted that with this implementation the power can be ramped very quickly by a rate of about $10 \%$ minute. This example shows that a HELIAS power plant could be operated with a flexibility in the range of $\pm 20 \%$ $( \pm 250 \mathrm{MW})$, which is essentially limited by the scale range of the turbine.

It should be emphasised that the scenario presented here is just an example. In reality, a dedicated scenario will depend on the stakeholder and the electricity demand / market. However, given the storage capabilities and a grid demand forecast, a runtime scenario can be planned ahead of time in a very flexible manner reacting to day-to-day circumstances.

\section{Conclusions and Outlook}

A conceptual design for a flexible HELIAS BoP for the $\mathrm{HCPB} \mathrm{BB}$ has been presented in this work. In alignment with the symmetry of a five-field-period HELIAS, 5 IB and 10 OB heat transfer loops are considered. Between the PHTS and PCS, an IHTS has been introduced including an ESS consisting of an industrial solar salt 2-tank design commonly found in CSP plants. The PCS is a classical water-steam Rankine cycle. generator which boils and superheats the feedwater which is then delivered to the turbine for electricity generation leading to a gross efficiency of about $38-39 \%$.

In absence of a detailed design for the HCPB BB in stellarator geometry and the ex-vessel piping, the pressure drop along the heat transfer loops had to be estimated. As nominal value, the result from DEMO calculations was used. However, due to 
the sensitivity of the required pumping power on the pressure drop, a sensitivity study was carried out which showed that even small changes in the pressure drop significantly impacts the pumping power.

Finally, an exemplary flexible runtime scenario was presented where $10 \%$ of the nominal power was stored as high-grade heat ${ }_{365}$ in the ESS. This allowed to increase the electrical power to $120 \%$ of the nominal value during a high demand time. While this scenario was just an example, it demonstrates the capability of a stellarator fusion power plant to operate very flexibly ${ }^{370}$ $( \pm 20 \%)$ in an envisaged dynamic future energy system.

Considering demand forecasts and load following mechanisms, a stellarator fusion power plant (FPP) seems well equipped to cope with fluctuating grid demands within its technical limitations. If the conceptual idea for the HELIAS BoP as presented in this paper can be confirmed by more detailed technical studies, then a stellarator FPP could potentially contribute to grid stabilization in a future energy system while replacing fossil fuel power plants as a sustainable energy source

\section{Acknowledgments}

The authors would like to express their appreciation for the fruitful discussions with Wolfgang Hering (KIT).

This work has been carried out within the framework of the EUROfusion Consortium and has received funding from the Euratom research and training programme 2014-2018 under grant agreement No 633053. The views and opinions expressed herein do not necessarily reflect those of the European Commission.

This work was also supported by the Helmholtz Association under the Joint Initiative "Energy System 2050 - A Contribution of the Research Field: Energy" as well as by the Initiative and Networking Fund of the Helmholtz Association eV.

\section{References}

[1] F. Romanelli, L. H. Federici, R. Neu et al. "A roadmap to the realization of fusion energy." Proc. IEEE 25th Symp. Fusion Eng, pp. 1-4 (2013)

[2] E. Bubelis, W. Hering and S. Perez-Martin. "Conceptual designs of PHTS, ESS and PCS for DEMO BoP with helium cooled BB concept." Fusion Engineering and Design (2018).

[3] F. Hernández, P. Pereslavtsev, Q. Kang et al. "A new HCPB breeding blanket for the EU DEMO: Evolution, rationale and preliminary performances." Fusion Engineering and Design, vol. 124, pp. 882 (2017). Proceedings of the 29th Symposium on Fusion Technology (SOFT-29) Prague, Czech Republic, September 5-9, 2016.

[4] F. Schauer, K. Egorov and V. Bykov. "HELIAS 5-B magnet system structure and maintenance concept." Fusion Engineering and Design, vol. 88, p. 1619 (2013).

[5] F. Warmer, S. Torrisi, C. D. Beidler et al. "Systems Code Analysis of HELIAS-type Fusion Reactor and Economic Comparison to Tokamaks." IEEE Transactions on Plasma Science, vol. 44, pp. 1576 (2016).

[6] I. Moscato, L. Barucca, S. Ciattaglia et al. "Preliminary design of EU DEMO helium-cooled breeding blanket primary heat transfer system." Fusion Engineering and Design (2018).

[7] L. Barucca, S. Ciattaglia, M. Chantant et al. "Status of EU DEMO heat transport and power conversion systems." Fusion Engineering and Design (2018).

[8] P. Pereslavtsev, U. Fischer, F. Hernandez et al. "Neutronic analyses for the optimization of the advanced HCPB breeder blanket design for DEMO." Fusion Engineering and Design, vol. 124, pp. 910 (2017). Proceedings of the 29th Symposium on Fusion Technology (SOFT-29) Prague, Czech Republic, September 5-9, 2016.

[9] F. Warmer and A. Häußler. "Estimation of Thermal Loads for a HELIAS power plant." submitted to Fusion Engineering and Design (2018).

[10] F. Warmer, V. Bykov, M. Drevlak et al. "From W7-X to a HELIAS fusion power plant: On engineering considerations for next-step stellarator devices." Fusion Engineering and Design, vol. 123, pp. 47 (2017). Proceedings of the 29th Symposium on Fusion Technology (SOFT-29) Prague, Czech Republic, September 5-9, 2016.

[11] F. Warmer, C. D. Beidler, A. Dinklage et al. "From W7-X to a HELIAS fusion power plant: motivation and options for an intermediate-step burning-plasma stellarator." Plasma Physics and Controlled Fusion, vol. 58, no. 7, p. 074006 (2016).

12] R. I. Dunn, P. J. Hearps and M. N. Wright. "Molten-Salt Power Towers: Newly Commercial Concentrating Solar Storage." Proceedings of the IEEE, vol. 100, no. 2, pp. 504 (2012).

[13] STEAG System Technologies. "EBSILON Professional, https://www.steag-systemtechnologies.com/de/produkte/ebsilonprofessional/." (2019). 